\title{
Scleral Buckling with Viscoelastics or Gas Injection for Bulging Retinal Detachments: A Retrospective Cohort Study
}

\author{
Quan-Yong Yi (D), Wen-Die Li, Qian Gui, Sang-Sang Wang, Li-Shuang Chen, Jing-Hai Mao, \\ Yan Gong, and Xia-Jun Wang
}

Ningbo Eye Hospital, Ningbo, China

Correspondence should be addressed to Quan-Yong Yi; quanyong_yi@163.com

Received 18 January 2021; Revised 20 March 2021; Accepted 30 March 2021; Published 8 April 2021

Academic Editor: Alessandro Meduri

Copyright ( 2021 Quan-Yong Yi et al. This is an open access article distributed under the Creative Commons Attribution License, which permits unrestricted use, distribution, and reproduction in any medium, provided the original work is properly cited.

\begin{abstract}
Objective. To examine the use of a viscoelastic agent instead of air in the vitreous cavity during surgery for scleral buckling. Methods. This was a retrospective cohort study of patients who underwent scleral buckling surgery for bulging rhegmatogenous retinal detachment (RRD) at Ningbo Eye Hospital from 07/2016 to 12/2019. The patients were grouped into drainage, air injection, cryotherapy and explant (DACE) and drainage, viscoelastic injection, cryotherapy, and explant (DVCE) groups, which were comparatively assessed. Results. There were 25 and 22 patients in the DVCE and DACE groups, respectively. The surgery was significantly shorter with DVCE than DACE $(P<0.05)$, with less intraoperative external pressure adjustment $(P<0.05)$. BCVA was lower in the DVCE group at 1 week compared with the DACE group $(P<0.05)$. Successful retinal reattachment was observed in $92.0 \%$ and $81.8 \%$ of the DVCE and DACE groups, respectively $(P<0.05)$. Cases requiring laser replenishing after the operation were less in the DVCE group compared with the DACE group $(P<0.05)$. There were no differences in complications and intraocular pressure between the two groups (all $P<0.05$ ). Conclusion. DVCE has better operative characteristics and faster vision recovery than DACE, with similar outcomes.
\end{abstract}

\section{Introduction}

Retinal detachment is the separation of the neurosensory retina from the underlying retinal pigment epithelium $[1,2]$. Primary retinal detachment refers to rhegmatogenous retinal detachment (RRD) that occurs due to holes, tears, or breaks in the retina of a previously uninvolved eye with the lens present without complicating factors (e.g., underlying diabetic retinopathy and penetrating trauma) or cataract surgery performed, usually with artificial intraocular lens $[1,3]$. RRD affects one in 10,000 individuals annually $[1,2]$, with an estimated lifetime risk of $3 \%$ by 85 years of age [1].

The key treatment of RRD is to close the hole. Many RRD cases can be managed successfully without subretinal fluid (SRF) drainage, and scleral buckling surgery is the classic treatment approach for RRD [4,5]. For bulging retinal detachment with a high degree of retinal bulge, subretinal fluid release is still required to promote retinal reattachment and accurately locate the hole perioperatively. Nevertheless, in traditional buckling surgery, after releasing the subretinal fluid, intraocular pressure decreases, and it is necessary to inject gas into the vitreous cavity for increasing the intraocular pressure to complete the operation. For scleral buckling surgery of highly bulging retinal detachment, there are two classic surgical methods. The first is cryotherapy, drain, air injection, and explant (CDAE). This method has two shortcomings: (1) as the bulging retina sometimes has a high degree of swelling, the gap between the choroid and the retina is wide when the fluid is not discharged, which makes it impossible to perform smooth condensation and positioning; (2) liquid discharge after condensation increases the risk of subchoroidal bleeding. The second method for bulging retinal detachment is drainage, air injection, cryotherapy, and explant (DACE). This method consists of draining the subretinal fluid first, which leads to retinal reattachment, and then injecting air to maintain intraocular pressure to complete the operation $[6,7]$. However, it also has some shortcomings: after injecting air, the density and 
refractive index of the air are quite different from those of the vitreous in the eye, which affects the observation of subsequent operation steps such as retinal tear positioning and condensation $[8,9]$. Based on the above shortcomings, novel and effective approaches are needed for scleral buckling surgery of highly bulging retinal detachment.

Viscoelastic agents are commonly used as auxiliary materials in ophthalmic surgery, especially cataract and vitreous surgery [10-12]. Comparing phacoemulsification surgery (PE) with viscosurgical devices (OVDs) and without OVD, best-corrected visual acuity (BCVA) values were significantly different on the day following surgery $(0.41 \pm 0.26$ and $0.54 \pm 0.34 \operatorname{logMAR})$ in OVD and non-OVD groups, respectively, although both groups had similar values at 6 months postoperatively [12]. The density and refractive index of a viscoelastic agent are basically close to those of the vitreous [13-15]. Recently, local dry vitrectomy combined with segmental scleral buckling and viscoelastic tamponade was evaluated for treating partial rhegmatogenous retinal detachment (RRD) with local vitreous traction, and postoperative visual acuities showed improvement or remained stable in the totality of patients, with no major complications besides transient mild intraocular pressure increased in 3 of 11 patients [16]. Accordingly, we hypothesized that, unlike air, a given viscoelastic agent should have no or little effects on the location of the hole and the condensation treatment.

Therefore, this study aimed to examine whether the use of a viscoelastic agent instead of air in the vitreous cavity during surgery for scleral buckling could achieve good outcomes. We found that drainage, viscoelastic injection, cryotherapy, and explant (DVCE) (Figure 1) is superior to DACE in terms of operative characteristics, allowing faster vision recovery with similar outcomes in the management of RRD.

\section{Materials and Methods}

2.1. Study Design. This was a retrospective cohort study of patients who underwent scleral buckling surgery for bulging RRD at Ningbo Eye Hospital from July 2016 to December 2019. This study complied with the Declaration of Helsinki and was reviewed and approved by the Ethics Committee of Ningbo Eye Hospital (no. 20200311).

Inclusion criteria were as follows: (1) bulging RRD; (2) RRD with equatorial and anterior hiatus requiring scleral buckling surgery; (3) bulging RRD requiring SRF drainage; (4) vitreous in good condition (no bleeding or severe turbidity); (5) proliferative vitreoretinopathy (PVR) grades A to $\mathrm{C}$; and (6) hole size of 1 to $4 \mathrm{DD}$, but with the possibility to close the lower serrated edge to 1 quadrant. Exclusion criteria were as follows: (1) planned vitrectomy or a history of vitreoretinal surgery; (2) fundus not seen due to severe turbidity of the refractive interstitium; (3) a history of uveitis; and (4) a history of trauma.

2.2. Preoperative and Postoperative Examinations. All patients underwent naked eye vision, corrected vision, intraocular pressure, refractive status, slit lamp microscope, fundus, Aalborg photography, eye B-mode ultrasound, and optical coherence tomography (OCT) examinations. Break location and number and the extent of peripheral retina and macular detachment were determined by front lens, trimirror, and indirect ophthalmoscopy. During the operation, surgery time (from the cutting of the conjunctiva to the final suture), the position of the scleral puncture, use of cerclage as well as silica gel and silicone sponge, and anesthesia were recorded.

BCVA was measured by the international standard visual acuity chart. For statistical analysis, BCVA was converted into logarithm of the minimum angle of resolution (LogMAR) values. $\operatorname{LogMAR}=\lg (1 /$ decimal visual acuity). The index was converted to 1.86; the manual conversion was 2.28 , and light perception was converted to 2.84 .

2.3. Surgical Process. The ophthalmologists discussed with the patient about the pros and cons of DACE and DVCE, and the surgical approach was finally selected by the patient. The two surgical procedures were similar, except that DACE used air for injection, while DVCE used viscoelastic injection (Figure 1).

Before the operation, all patients underwent full mydriasis with Medori eye drops and 1\% atropine eye drops, followed by $2 \%$ lidocaine and $0.75 \%$ bupivacaine for anesthesia. All operations were performed by the same surgeon (Dr. Y.Q.Y). All patients underwent scleral buckling surgery. Before surgery, systemic and eye infections were ruled out and controlled, and blood glucose and blood pressure were controlled. A HEINI indirect ophthalmoscope, a Suzhou Xinmingren carbon dioxide condenser, a silica gel strip, a silica gel block, and a silicon sponge were used. After routine disinfection and anesthesia, according to hiatus' position and the scope of external compression, the bulbar conjunctiva was cut along the corneoscleral limbus. The corresponding extraocular rectus muscles were separated and pulled.

For DVCE, a 27-G needle was used to puncture the scleral wall at a 30-degree angle at the highest point of the retinal bulge. The depth was based on the feeling of the puncture and outflow of SRF. A retractor was used for pressing the eyeball wall to apply an appropriate pressure to maintain intraocular pressure and facilitate the outflow of SRF from the puncture port (Figure 2(a)). After draining the SRF, the vitreous cavity was punctured $4 \mathrm{~mm}$ behind the limbus of the cornea to inject the viscoelastic agent (VisCoat, Alcon, Hunenberg, Switzerland) to the intraocular pressure $\mathrm{T}_{-1}$ (Figure 2(b)). For DACE, all steps were the same as described for DVCE, except that air was injected instead of the viscoelastic agent. After draining the SRF, the vitreous cavity was punctured $4 \mathrm{~mm}$ behind the limbus of the cornea, and sterile air was injected into the intraocular pressure $T_{n}$.

After injection of the viscoelastic agent or sterile air, a $\mathrm{CO}_{2}$ condensing pen was used to press and condense the edge of the hole with live observation under an indirect ophthalmoscope. When the retina at the edge of the hole became white, the condensation process was stopped, and 


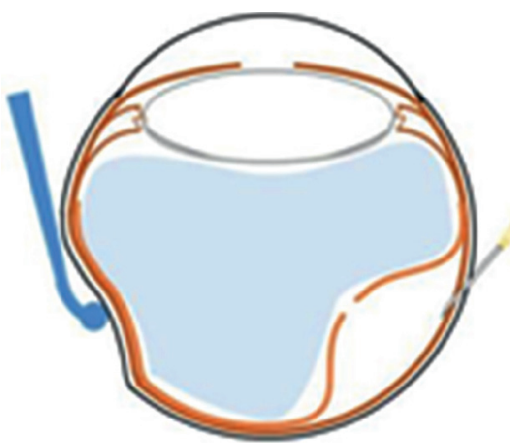

(a)

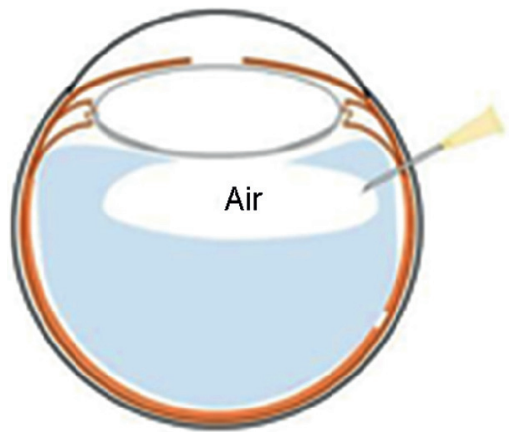

(b)

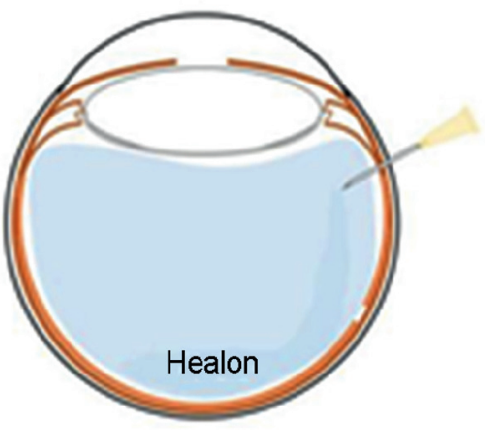

(c)

FIGURE 1: Schematic diagram of extrascleral drainage (a), air injection into the vitreous cavity (b), and viscoelastic agent injection into the vitreous cavity (c).

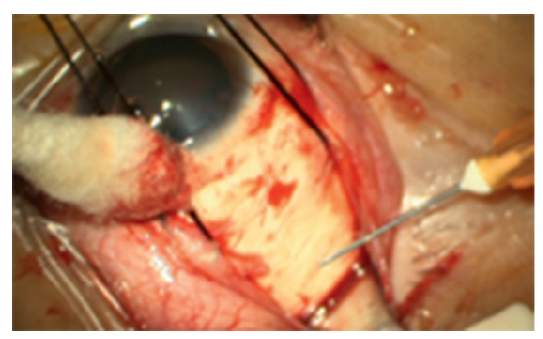

(a)

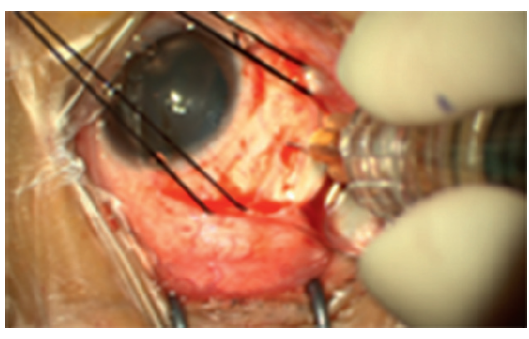

(b)

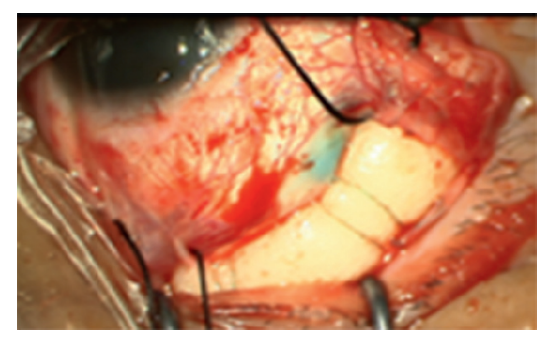

(c)

Figure 2: DVCE procedure. (a) Drainage. Puncture and drainage of the scleral wall corresponding to the highest point of the retinal bulge. (b) Injection of the viscoelastic agent. After supplying the subretinal fluid, a puncture was made $4 \mathrm{~mm}$ behind the limbus of the cornea and into the vitreous cavity to inject the viscoelastic agent for increasing intraocular pressure. (c) External pressure (explant). A suitable silicon sponge block or silicone tape was placed at the position of the hole.

trypan blue was applied. A suitable silicon sponge block or circling silicone tape as an explant was sewn at the position marked by the puncture (Figure 2(c)).

At the end of all the surgical procedures, dexamethasone $(5 \mathrm{mg} / 1 \mathrm{~mL})$ was injected into the inferior subconjunctival space. During follow-up, antiglaucoma eye drops such as $\beta$-blockers, carbonic anhydrase inhibitors, and prostaglandin analogs, were prescribed with IOP above $21 \mathrm{mmHg}$.

2.4. Outcome and Follow-Up. On the day after the operation, retinal reattachment, vision, and intraocular pressure were assessed. Complications were recorded: hole not closed by 1 week to 1 month after the operation, remaining SRF, unresolved retina detachment, and subretinal hemorrhage (subretinal distribution and size graded as less or greater than 1 disk area $(<1 \mathrm{DA}$ or $>1 \mathrm{DA}))$. BCVA, slit lamp microscopy findings, intraocular pressure, fundus examination using the Optomap panoramic eye scan system, and OCT data were reviewed at 1 week, 1 month, 3 months, and 6 months after surgery.

2.5. Statistical Analysis. Statistical analysis was performed with SPSS 17.0 (SPSS Inc., Chicago, USA). Normally and nonnormally distributed continuous variables were presented as mean \pm standard deviation (SD) and median (range), respectively, and compared by Student's $t$-test and the Mann-Whitney $U$-test, respectively. Categorical data were presented as $n(\%)$ and assessed by the chi-square test or Fisher's exact test, as appropriate. Two-sided $P<0.05$ was considered statistically significant.

\section{Results}

3.1. Characteristics of the Patients. There were 25 patients in the DVCE group (25 eyes), including 17 males and eight females. There were 22 patients (22 eyes) in the DACE group, including 15 males and seven females. The patient characteristics are presented in Table 1 . There were no significant differences between the two groups in age, sex, disease course, BCVA, and affected quadrants.

3.2. Intraoperative Characteristics. The surgery was significantly shorter with DVCE than DACE $(38 \pm 16$ vs. $49 \pm 15 \mathrm{~min}, P<0.05$ ) (Table 2). Intraoperative excessive pressure adjustment was observed in one patient of the DACE group and four patients of the DVCE group $(P<0.05)$. There were no significant differences in combined cerclage and subretinal hemorrhage (both $P<0.05$ ). There were no iatrogenic retinal injuries.

3.3. BCVA after Surgery. Table 3 presents BCVA values after surgery. BCVA was lower in the DVCE group at 1 week compared with the DACE group ( $1.5 \pm 0.5$ vs. $2.0 \pm 0.5$, 
TABLE 1: Characteristics of the patients.

\begin{tabular}{|c|c|c|c|}
\hline Parameters & DVCE & DACE & $P$ \\
\hline Cases, $n$ & 25 & 22 & \\
\hline Age, mean $\pm S D$, years & $36.16 \pm 16.23$ & $33.39 \pm 15.97$ & 0.749 \\
\hline Sex (male/female) & $17 / 8$ & $15 / 7$ & 0.813 \\
\hline Disease course, mean $\pm S D$, months & $3.12 \pm 1.12$ & $3.65 \pm 1.49$ & 0.376 \\
\hline Preoperative BCVA, logMAR \pm SD & $1.26 \pm 0.78$ & $1.39 \pm 0.67$ & 0.487 \\
\hline Intraocular pressure, median (range), $\mathrm{mmHg}$ & $6-20$ & $6-22$ & 0.429 \\
\hline \multicolumn{4}{|c|}{ Average number of quadrants of retinal detachment range, $n(\%)$} \\
\hline 1 quadrant & $16(64.0)$ & $14(63.6)$ & 0.725 \\
\hline $2-3$ quadrants & $7(28.0)$ & $7(31.8)$ & 0.481 \\
\hline All 4 quadrants & $2(8.0)$ & $1(4.5)$ & 0.396 \\
\hline Macular detachment, $n(\%)$ & $15(60.0)$ & $15(68.2)$ & 0.557 \\
\hline With lattice degeneration and cystic degeneration, $n(\%)$ & $17(68.0)$ & $15(68.2)$ & 0.891 \\
\hline
\end{tabular}

BCVA: best-corrected visual acuity; logMAR: logarithm of the minimal angle of resolution; DVCE: drainage, viscoelastic injection, cryotherapy, and explant; DACE: drainage, air injection, cryotherapy, and explant.

TABLE 2: Intraoperative characteristics.

\begin{tabular}{|c|c|c|c|}
\hline Parameters & DVCE $(n=25)$ & DACE $(n=22)$ & $P$ \\
\hline Operation time, mean $\pm \mathrm{SD}$, min & $38 \pm 16$ & $49 \pm 15$ & $<0.05$ \\
\hline Combined cerclage, $n(\%)$ & $2(8.0 \%)$ & $2(9.1 \%)$ & 0.965 \\
\hline Intraoperative external pressure adjustment, $n(\%)$ & $1(4.0 \%)$ & $4(18.2 \%)$ & $<0.05$ \\
\hline Subretinal hemorrhage, $n(\%)$ & $1(4.0 \%)$ & $1(4.5 \%)$ & 0.891 \\
\hline Iatrogenic retinal hole, $n(\%)$ & 0 & 0 & 0.875 \\
\hline
\end{tabular}

DVCE: drainage, viscoelastic injection, cryotherapy, and explant; DACE: drainage, air injection, cryotherapy, and explant.

$P=0.002)$. There were no significant differences in BCVA between the DVCE and DACE groups at 1,3, and 6 months (all $P<0.05$ ).

3.4. Follow-Up. There were no differences in IOP between the two groups at 1 week and 1,3 , and 6 months (all $P<0.05$ ) (Table 4). Successful retinal reattachment was observed in $92 \%$ and $82 \%$ patients of the DVCE and DACE groups, respectively $(P<0.05)$. Cases requiring laser replenishing after the operation were less in the DVCE group compared with the DACE group $(P<0.05)$. There were no differences in reoperation rate between the two groups.

\section{Discussion}

The use of air in the classical DACE surgery for RRD has disadvantages associated with the difference in refractive index between air and vitreous, and the gas injected into the vitreous cavity, which is usually located behind the lens, also affects observation [8,9]. Therefore, this study aimed to examine the use of a viscoelastic agent instead of air in the vitreous cavity during surgery for scleral buckling. The results suggested that DVCE has better operative characteristics and faster vision recovery than DACE, but similar outcomes, suggesting that viscoelastic agent use may be a new option for the treatment of bulging RRD.

The retinal hole is the main cause of retinal detachment, and the purpose of curing retinal detachment can be achieved by sealing the hole [17]. Although the related surgical methods have been continuously improved, the basic principle remains to close the hiatus, with minimal trauma, low economic cost, and relatively simple operation. Scleral buckle
TABLE 3: Best-corrected visual acuity after the operation.

\begin{tabular}{lcccc}
\hline & 1 week & 1 month & 3 months & 6 months \\
\hline $\begin{array}{l}\text { DVCE group } \\
\text { BCVA, }\end{array}$ & & & & \\
logMAR \pm SD & $1.50 \pm 0.45$ & $0.89 \pm 0.57$ & $0.68 \pm 0.61$ & $0.59 \pm 0.46$ \\
\hline $\begin{array}{l}\text { DACE group } \\
\text { BCVA, }\end{array}$ & $2.0 \pm 0.52$ & $0.95 \pm 0.61$ & $0.65 \pm 0.51$ & $0.62 \pm 0.49$ \\
logMAR \pm SD & $<0.05$ & 0.219 & 0.746 & 0.482 \\
$P$ & & & & \\
\hline $\begin{array}{l}\text { BCVA: best-corrected visual acuity; logMAR: logarithm of the minimal } \\
\text { angle of resolution; DVCE: drainage, viscoelastic injection, cryotherapy, } \\
\text { and explant; DACE: drainage, air injection, cryotherapy, and explant. }\end{array}$
\end{tabular}

surgery has been applied in clinical practice for nearly a hundred years because of minimal trauma, low cost, good curative effect, and few postoperative complications. For bulging retinal detachment, it is necessary to remove the SRF to facilitate retinal reattachment and condensation. On the other hand, removing the SRF decreases intraocular pressure, and injection of auxiliary substances into the vitreous cavity is therefore required to maintain intraocular pressure and to apply pressure on the retina to facilitate reattachment $[6,7]$. Previously, the use of perfluoroalkanes has been described [18]. The traditional scleral buckling surgery generally uses sterile air into the vitreous. However, the density and refractive index of air are quite different from those of the vitreous in the eye, which could affect the observation of subsequent operations such as retinal hole positioning and condensation; this might lead to complications or lower the success rate of the operation. Indeed, cataract, secondary glaucoma, and retinal artery occlusion have been reported 
TABle 4: Postoperative characteristics.

\begin{tabular}{lcc}
\hline Parameters & DVCE $(n=25)$ & DACE $(n=22)$ \\
\hline Intraocular pressure & & $P$ \\
1 week, median (range), mmHg & $16.5(6-38)$ & $16.2(7-36)$ \\
1 month, median (range), mmHg & $15.9(11-23)$ & $15.7(11-22)$ \\
3 months, median (range), mmHg & $17.1(9-23)$ & $17.4(11-24)$ \\
6 months, median (range), mmHg & $15.9(9-21)$ & $16.1(10-22)$ \\
Successful retinal reattachment, $n(\%)$ & $23(92.0 \%)$ & $18(81.8 \%)$ \\
Retinal reattachment failure, $n(\%)$ & $2(8.0 \%)$ & $4(18.2 \%)$ \\
New holes & 0 & $2(9.1 \%)$ \\
Missed holes & $1(4.0 \%)$ & $1(4.5 \%)$ \\
Deviation of external pressure & $1(4.0 \%)$ & $1(4.5 \%)$ \\
Scleral buckling again, $n(\%)$ & $1(4.0 \%)$ & $3(13.6 \%)$ \\
Vitrectomy again, $n$ (\%) & $1(4.0 \%)$ & $1(4.5 \%)$ \\
Need to replenish laser after the operation, $n(\%)$ & $4(16.0 \%)$ & $4(18.2 \%)$ \\
\hline
\end{tabular}

DVCE: drainage, viscoelastic injection, cryotherapy, and explant; DACE: drainage, air injection, cryotherapy, and explant.

with the use of gas $[19,20]$. In addition, when the patient switches from the supine position to the upright position after the operation, the gas in the vitreous cavity moves upward, which pulls the lower vitreous and the retina, potentially generating new holes. Newly generated tears are one of the causes of failure after scleral buckling surgery. In the DACE group in this study, two of the four unsuccessful retina reattachment cases were due to newly created inferior tears.

Viscoelastic agents are commonly used as auxiliary materials in clinical ophthalmic surgery, including cataract and vitreous surgery [10-12]. The density and refractive index of a viscoelastic agent is close to those of the vitreous body [10-12], which allows a more precise observation of the subsequent hole positioning and condensation treatment after viscoelastic agent injection into the vitreous cavity. In addition, viscoelastic agents can liquefy and are gradually absorbed as the vitreous regenerates, and do not stay long in the eye [10,21-23]. In this study, the operation time was significantly shorter in the DVCE group compared with the DACE group, probably because of better visualization and easier positioning. In addition, the patients in the DVCE group had better visual acuity at 1 week, but the difference was no more significant by 1 month. A previous study showed early visual recovery after using a viscoelastic agent, supporting the present findings [24]. In addition, a recent comparative multicenter study demonstrated that limited vitrectomy is time-efficient and effective in removing epiretinal membrane without additional complications compared to complete vitrectomy [25]. Another study reported good outcomes of sub-perfluoro- $n$-octane injection as a viscoelastic agent in patients with macular hole retinal detachment [26]. The rate of retinal reattachment was numerically higher, but the difference was not significant, probably because of the small number of patients. Animal studies showed high levels of anatomic reattachment after using a viscoelastic solution, supporting the present findings [14, 27, 28].

This study had limitations. It was a retrospective study, thus limited to the data available in medical charts. In addition, the sample size was small. Furthermore, different viscoelastic agents are available $[24,29,30]$, and future studies could be designed to compare them. Moreover, a retrospective study recently showed that microscope-assisted $a b$ externo surgery is effective and safe, reducing discomfort, allowing the surgeon to work with both hands free, and providing an optimal visualization of various surgical steps [25], which should be compared with the currently proposed approach in large multicenter prospective trials. Finally, follow-up was short, and the longterm effect of the viscoelastic agent should be examined.

\section{Conclusions}

In conclusion, DVCE has better operative characteristics and faster vision recovery than DACE, but similar outcomes, suggesting that viscoelastic agents for the treatment of bulging RRD have advantages over air. Additional studies are required to determine the optimal viscoelastic agent.

\section{Data Availability}

No datasets were generated for this study.

\section{Conflicts of Interest}

The authors declare that there are no conflicts of interest regarding the publication of this paper.

\section{Acknowledgments}

This study was funded by Zhejiang Provincial Medicine and Health Science and Technology Project (no. 2018KY735), Ningbo Natural Science Foundation (no. 2019A610351), Ningbo Yinzhou District Science and Technology Plan Project ([2017] no. 59 and [2018] no. 108).

\section{References}

[1] H. K. Kang and A. J. Luff, "Management of retinal detachment: a guide for non-ophthalmologists," British Medical Journal, vol. 336, no. 7655, pp. 1235-1240, 2008.

[2] R. F. Gariano and C. H. Kim, "Evaluation and management of suspected retinal detachment," American Family Physician, vol. 69, no. 7, pp. 1691-1698, 2004.

[3] D. J. D'Amico, "Primary retinal detachment," New England Journal of Medicine, vol. 359, no. 22, pp. 2346-2354, 2008. 
[4] S. Blindbaek and J. Grauslund, "Prophylactic treatment of retinal breaks-a systematic review," Acta Ophthalmologica, vol. 93, no. 1, pp. 3-8, 2015.

[5] C. D. Poulsen, T. Peto, J. Grauslund, and A. Green, "Epidemiologic characteristics of retinal detachment surgery at a specialized unit in Denmark," Acta Ophthalmologica, vol. 94, no. 6 , pp. $548-555,2016$.

[6] C. Gilbert and D. McLeod, "D-ACE surgical sequence for selected bullous retinal detachments," British Journal of Ophthalmology, vol. 69, no. 10, pp. 733-736, 1985.

[7] I. A. Pearce, D. Wong, J. McGalliard, and C. Groenewald, "Does cryotherapy before drainage increase the risk of intraocular haemorrhage and affect outcome? a prospective, randomised, controlled study using a needle drainage technique and sustained ocular compression," British Journal of Ophthalmology, vol. 81, no. 7, pp. 563-567, 1997.

[8] M. Saeed, R. Ganeshalingham, and D. McHugh, "PaCE: a technique to avoid subretinal fluid drainage in retinal detachment surgery," Acta Ophthalmologica, vol. 84, no. 1, pp. 47-53, 2006.

[9] K. A. M. Solaiman, M. A. Alaswad, A. Mahrous et al., "Drain fluid cryo-explant technique for treatment of superior bullous rhegmatogenous retinal detachment," Therapeutic Advances in Ophthalmology, 2020.

[10] T. Higashide and K. Sugiyama, "Use of viscoelastic substance in ophthalmic surgery-focus on sodium hyaluronate," Clinical Ophthalmology, vol. 2, no. 1, pp. 21-30, 2008.

[11] A. Mitani, T. Suzuki, Y. Tasaka et al., "Evaluation of a new method of irrigation and aspiration for removal of ophthalmic viscoelastic device during cataract surgery in a porcine model," BMC Ophthalmology, vol. 14, p. 129, 2014.

[12] I. Taskin and L. Aslan, "Effects of phacoemulsification with versus without viscoelastic devices on surgical outcomes," International Ophthalmology, vol. 38, no. 1, pp. 5-10, 2018.

[13] S. Donati, S. M. Caprani, G. Airaghi et al., "Vitreous substitutes: the present and the future," Biomed Research International, vol. 2014, no. 3, 12 pages, Article ID 351804, 2014.

[14] C. D. Pritchard, S. Crafoord, S. Andréasson et al., "Evaluation of viscoelastic poly (ethylene glycol) sols as vitreous substitutes in an experimental vitrectomy model in rabbits," Acta Biomaterialia, vol. 7, no. 3, pp. 936-943, 2011.

[15] N. K. Tram, P. Jiang, T. C. Torres-Flores, K. M. Jacobs, H. L. Chandler, and K. E. Swindle-Reilly, "A hydrogel vitreous substitute that releases antioxidant," Macromolecular Bioscience, vol. 20, no. 2, Article ID e1900305, 2020.

[16] P. Fei, H. Y. Jin, Q. Zhang et al., "Local dry vitrectomy combined with segmental scleral buckling and viscoelastic tamponade for rhegmatogenous retinal detachment with vitreous traction," International Journal of Ophthalmology, vol. 13, no. 11, pp. 1713-1719, 2020.

[17] B. P. Gloor and M. F. Marmor, "Controversy over the etiology and therapy of retinal detachment: the struggles of Jules Gonin," Survey of Ophthalmology, vol. 58, no. 2, pp. 184-195, 2013.

[18] C. Zhou, Q. Qiu, and Z. Zheng, "Air versus gas tamponade in rhegmatogenous retinal detachment with inferior breaks after 23-gauge pars plana vitrectomy," Retina, vol. 35, no. 5, pp. 886-891, 2015.

[19] G. F. Hilton and P. E. Tornambe, "Pneumatic retinopexy. The retinal detachment study group," Retina, vol. 11, no. 3, pp. 285-294, 1991.

[20] G. F. Hilton, P. E. Tornambe, D. A. Brinton et al., "The complication of pneumatic retinopexy," Transactions of the American Ophthalmology Society, vol. 88, pp. 191-207, 1990.
[21] H. Bleckmann, R. Vogt, and H.-J. Garus, "Collagel-a new viscoelastic substance for ophthalmic surgery," Journal of Cataract and Refractive Surgery, vol. 18, no. 1, pp. 20-26, 1992.

[22] S. Kovacevic, Z. Pastar, Z. Sessa, V. Krpina, and E. Paic, "Comparison of the effect of two viscoelastic agents on an early postoperative intraocular pressure," Collegium Antropologicum, vol. 29, no. S1, pp. 115-117, 2005.

[23] G. Rainer, R. Menapace, O. Findl et al., "Intraocular pressure rise after small incision cataract surgery: a randomised intraindividual comparison of two dispersive viscoelastic agents," British Journal of Ophthalmology, vol. 85, no. 2, pp. 139-142, 2001.

[24] X. J. Ren, S. C. Bu, D. Wu et al., "Patching retinal breaks with healaflow in 27-gauge vitrectomy for the treatment of rhegmatogenous retinal detachment," Retina, vol. 40, no. 10, pp. 1900-1908, 2020.

[25] R. Frisina, M. Forlini, P. Date et al., "Microscope-assisted ab externo surgery for the treatment of primary rhegmatogenous retinal detachment-new tech meets old art," Ophthalmic Research, vol. 63, no. 1, pp. 34-40, 2020.

[26] C. C. Lai, A. L. Wu, H. D. Chou et al., "Sub-perfluoro-noctane injection of ocular viscoelastic device assisted inverted internal limiting membrane flap for macular hole retinal detachment surgery: a novel technique," BMC Ophthalmology, vol. 20, no. 1, p. 116, 2020.

[27] H. Barth, S. Crafoord, and F. Ghosh, "A new retinal detachment treatment model for evaluation of vitreous tamponades," Current Eye Research, vol. 46, no. 3, pp. 373-379, 2020.

[28] S. Crafoord, S. Andreasson, and F. Ghosh, "Experimental vitreous tamponade using polyalkylimide hydrogel," Graefe's Archive for Clinical and Experimental Ophthalmology, vol. 249, no. 8, pp. 1167-1174, 2011.

[29] K. Teruya, J. Sueda, M. Arai et al., "Patching retinal breaks with seprafilm in experimental rhegmatogenous retinal detachment of rabbit eyes," Eye, vol. 23, no. 12, pp. 2256-2259, 2009.

[30] J. P. Hubschman, A. Govetto, M. Farajzadeh, T. Sato, S. Askari, and B. Glasgow, "Feasibility of a polyethylene glycol-derived polymer as retinal patch to seal retinal breaks during vitrectomy for rhegmatogenous retinal detachment: a prospective, in vivo pilot study in a porcine model," Clinical \& Experimental Ophthalmology, vol. 45, no. 7, pp. 708-716, 2017. 